\title{
Advancing Cryptosporidium Diagnostics from Bench to Bedside
}

\author{
Raul V. Destura $^{1} \cdot$ Rohani B. Cena $^{1} \cdot$ Ma. Jowina H. Galarion ${ }^{1} \cdot$ Coleen M. Pangilinan $^{1}$ • \\ Geraldine M. Arevalo ${ }^{1}$ Ryan Oliver C. Alba ${ }^{2}$ - Joy Ann G. Petronio ${ }^{1}$ • \\ Gielenny M. Salem ${ }^{1}$ • Brian Schwem ${ }^{1}$ - Jesus Emmanuel A. D. Sevilleja ${ }^{1}$
}

Published online: 22 July 2015

(C) Springer International Publishing AG 2015

\begin{abstract}
Cryptosporidium is increasingly being recognized as an important cause of diarrhea worldwide. Although well known for its impact among HIV positive population, improved diagnostic tests have contributed to its emerging recognition one among the most prevalent causes of early childhood moderate to severe diarrhea, persistent diarrhea, and impaired neurocognitive development. The diagnosis of Cryptosporidiosis is generally carried out based on availability of skilled microscopist or advanced equipment for molecularand immunologic-based assays. As an emerging enteric pathogen of medical importance, the need for point-of-care technology is deemed necessary for early identification of the pathogen and application of infection control measures for its potential risk of creating outbreaks. Current point-of-care technologies demonstrate varying sensitivities and specificities and may already address the present diagnostic need.
\end{abstract}

This article is part of the Topical Collection on Protozoa

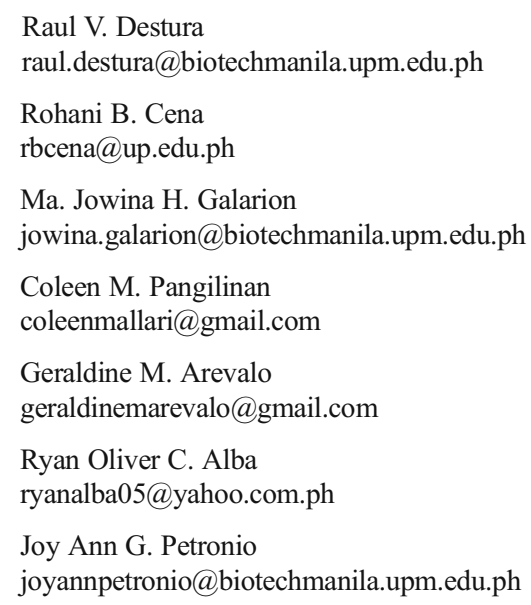

Keywords Cryptosporidium sp. · Diagnostics · Point-of-care

\section{Introduction}

\section{The Need for Sensitive Cryptosporidium Diagnostics}

The recent identification of cryptosporidiosis as an important cause of morbidity and mortality worldwide has been suggested to be due to gaps in diagnosis, treatment, and immunization $[1 \bullet, 2,3]$. This article will focus on conventional and innovative technologies employed for sensitive and rapid Cryptosporidium diagnostics.

Cryptosporidium is a protozoan that is increasingly being recognized as an important cause of diarrhea worldwide. Of the more than 26 Cryptosporidium species, Cryptosporidium
Gielenny M. Salem

gielennymsalem@yahoo.com.ph

Brian Schwem

beschwem@gmail.com

Jesus Emmanuel A. D. Sevilleja

jesusemmanuel.sevilleja@cantab.net

1 National Institute of Molecular Biology and Biotechnology, National Institutes of Health, University of the Philippines Manila, NIH Building, Pedro Gil St. Ermita, Manila 1000, Philippines

2 Office of the Vice Chancellor for Research, National Institutes of Health, University of the Philippines Manila, NIH Building, Pedro Gil St., Ermita, Manila 1000, Philippines 
parvum (humans and ruminants) and Cryptosporidium hominis (humans only) are the most important causes of human infections and are morphologically indistinguishable. All species of Cryptosporidium are obligate intracellular parasites that undergo development leading to the excretion of 4-6 $\mu \mathrm{m}$ sized oocysts in feces. Infected people can excrete oocysts for as long as 60 days after infection [4]. Transmission of Cryptosporidium is mainly through the oral-fecal route with an infectious dose as low as 10 oocysts [5]. The small size of Cryptosporidium, persistence of shedding, and low infective dose allow the parasite to easily spread in places with inadequate sanitation and hygiene. Even in resource abundant settings, the parasite can evade detection resulting in outbreaks, the largest of which was documented in Milwaukee, Wisconsin USA and accounted for more than 400,000 infections and 100 deaths [6].

Cryptosporidium sp. are ubiquitous and can be found in untreated drinking and recreational water, contaminated food, day care centers, and hospitals $[7,8]$. Exposure to farm animals and children, initially infected with this parasitic organism can lead to the further propagation of the organism. Through flooding and increased rainfall, climate change is predicted to increase the burden of waterborne diseases including Cryptosporidium [9]. The observations of esophageal cryptosporidiosis, where parasites were found in squamous mucosa and submucosal lumen and border [10], extraintestinal manifestations of Cryptosporidium infection in severely immunocompromised patients [11•], and respiratory cryptosporidiosis concurrent with intestinal cryptosporidiosis in HIV-seronegative children [12] suggest the potential for more diverse routes of transmission. With the possible increase in the burden of Cryptosporidium, there is a need to develop more sensitive diagnostic techniques to detect the organism in clinical, biological, and environmental specimens [7].

\section{Current Diagnostic Platforms}

\section{Serological and microscopic Diagnosis of Cryptosporidium}

Studies focusing on comparisons of the effectiveness and reliability of various methods in diagnosing Cryptosporidiosis greatly increased since its identification as a gastrointestinal and respiratory tract parasitic disease among many species of animals, including mammals, in the 1970s To date, there is no single ideal test for the diagnosis of Cryptosporidiosis, and a significant percentage of Cryptosporidium infections can be missed if any of the available assays has been the sole method of diagnosis [13] (Table 1). Thus, current research mainly focuses on determining the most sensitive and cost-effective diagnostic method that can detect infection even at the earliest stages before the parasite has multiplied. Such research aims to draw benefit from knowing the most reliable tool in detecting the presence of Cryptosporidium parasite in vulnerable population groups and its environmental reservoir which may lead to enhanced diagnostic surveillance and clinical tools for effective eradication measures of the disease.

One approach to detect Cryptosporidium infection is the serological detection of the specific antibody response. Following infection, there is usually the development of characteristic immunoglobulin $\mathrm{G}(\mathrm{IgG}), \operatorname{IgA}$, and IgM antibody response against 2 sporozoite surface antigens with apparent molecular masses of approximately 27,15 , and $17 \mathrm{kDa}$ [14•]. IgG responses to Cryptosporidium sporozoite antigens of low molecular size can persist for several months [1] in adults and have been shown to be consistent and of sufficient intensity to act as reliable markers of exposure $[15,16]$.

Prior studies suggested that serum Cryptosporidium-specific antibody levels remain elevated for weeks to months following infection [15]. Increases in the intensity of Western blot response for IgG antibody to two Cryptosporidium antigens (17 and $27 \mathrm{kDa})$ following infection were observed among highly exposed individuals. A $15 \mathrm{kDa}$ protein isolated from the feces of infected calves by immunoaffinity adsorption using a monoclonal anti $C$. parvum antibody was recognized by IgA antibodies present in the saliva during the convalescent phase of infection. These results suggest that this coproantigen may be released from C. parvum sporozoites and may induce IgA antibody production in the mucosal immune system of infected calves [17]. The seroprevalence of IgG antibodies to the $27-\mathrm{kDa}$ and $15 / 17-\mathrm{kDa}$ Cryptosporidium antigen in sera was significantly greater (49-61\%) in settlements where the drinking water originated from surface water than in the control city where riverbank filtration was used (21-23\%). Hence, the elevated responses were most likely due to the use of contaminated water [18•].

It is necessary to evaluate the comparative diagnostic accuracy and feasibility of newly introduced test in comparison to the existing tests or the criterion standard to reveal how well this test discriminates between health and disease. The Center for Disease Control and Prevention Project \#438 has developed a second generation ELISA that detected all laboratory confirmed cases. It showed improved sensitivity over the immunoblot test results [19]. There was also no cross-reactivity between Cryptosporidium and Giardia or Toxoplasma. Moreover, ELISA results from the same sample sets from two different laboratories showed excellent correlation. While these serological assays for Cryptosporidium are important for epidemiological studies because specific antibody responses develop after both symptomatic and asymptomatic infection, they cannot be used to diagnose active infection.

Cryptosporidium can only be cultivated for a short time in epithelial cell monolayers and does not grow in conventional laboratory media. Thus, microscopy is considered the conventional method of detecting active infection. Accuracy of diagnostic microscopy may be affected by several factors. The 


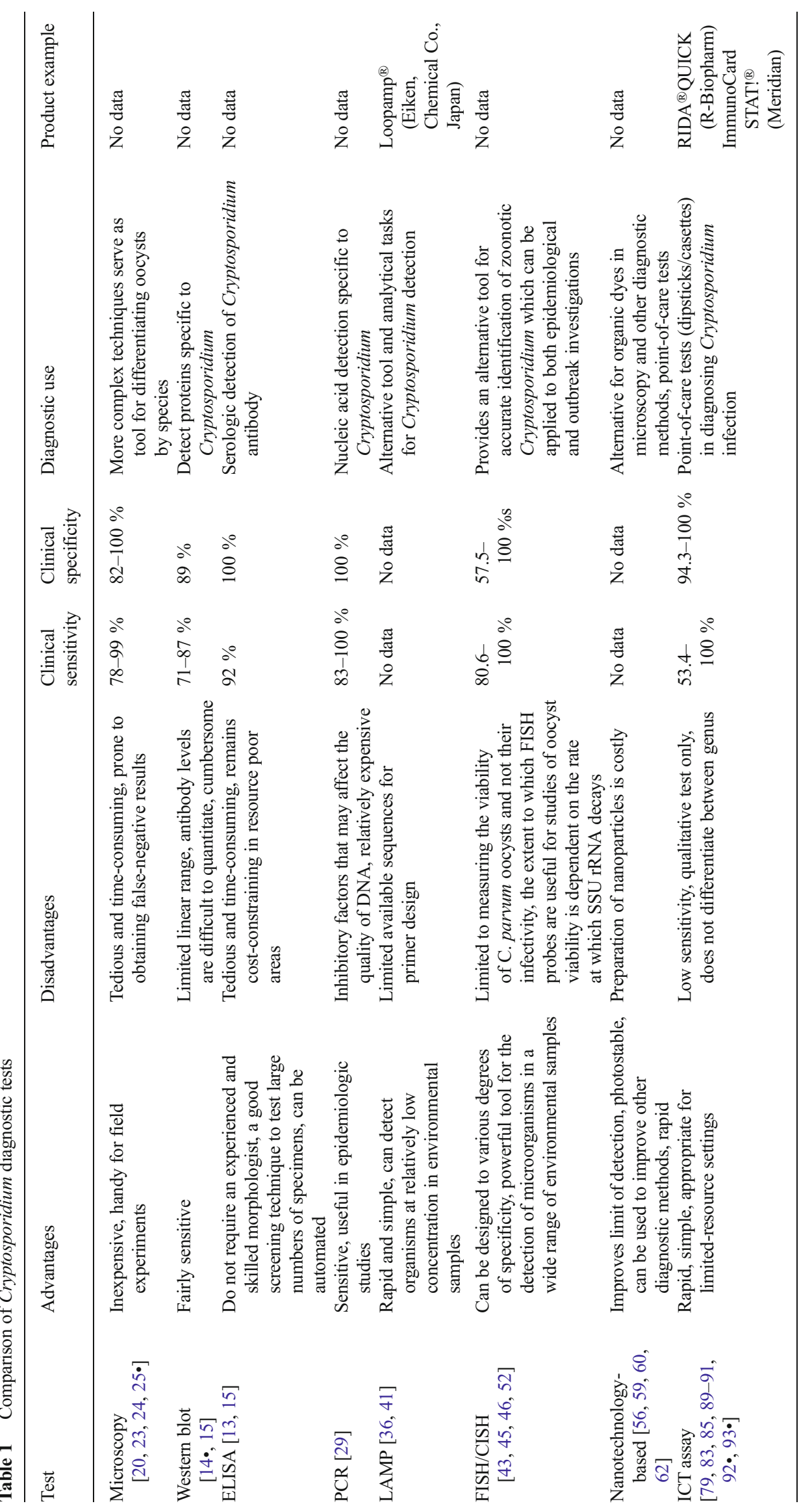


majority of positive Cryptosporidium samples are not usually found in watery stools, rather in loose mushy stools which implies that microscopy might not efficiently work for highly turbid samples as solid particles might cover the target organisms. Multiple staining and washing steps of the fecal sample on the slide could also lessen the detectable quantity of oocysts since oocysts can be washed away in the process [20]. The significant impact of the skills and experience in processing slides and analyzing stool samples of the microscopist is one limiting factor affecting accuracy of results. The generally low sensitivity results for microscopic diagnosis are usually augmented by performing repeated microscopic examination to confirm a microscopic finding. This basically implies that aside from being a less effective diagnostic tool compared to other serological techniques, conventional microscopy is also time-consuming and tedious, as it generally requires an average rate of $10 \mathrm{~min}$ per slide before oocysts can be detected and its diagnostic accuracy is largely dependent on the experience and skills of the microscopist [21•].

Several preparation and staining techniques were developed to improve detection of parasites in stool samples. Among the four existing commonly used preparation techniques for microscopic analysis of stool samples: Sheather's flotation (SF), normal saline sedimentation staining (NSSS), direct fecal smear staining (DFSS), and Sheather's flotation sedimentation staining (SFSS), the latter is considerably more sensitive and specific compared with the other three, having sensitivity and specificity values of 82.6 and $98.8 \%$, respectively [22]. Most laboratories use a modified acid-fast or Safranin stain to detect oocysts. Two other microscopic methods namely auramine phenol microscopy and immunofluorescence microscopy have improved sensitivity, 92.1 and $97.4 \%$, respectively, though it still has the potential to produce false-negative results [23]. Despite its downsides, the use of microscopy as a diagnostic tool for Cryptosporidium remains to be the most common technique to detect presence of active infection and is the only technique that can distinguish the presence of oocysts. However, when there is high index of suspicion, it is highly advisable to do confirmatory tests, such as PCR for microscopy-negative samples. While highly sensitive, molecular-based tests, such as polymerase chain reaction (PCR), enzyme-linked immunosorbent assay (ELISA), and some immunochromatographic tests such as the use of ImmunoCard STAT ${ }^{\circledR}[23,24,25 \cdot]$ are not routinely available in most laboratories.

In the clinical setting, there is a need for rapid, sensitive, and specific diagnostic methods that can guide appropriate control and therapy for cryptosporidiosis [23]. Antigenbased detection using ELISA and Western blot methods has been widely used. ELISA, however, has its limitations which involves the relative decrease specificity due to occurrence of cross reactions with closely related parasitic antigens. In addition, some comparative evaluation studies revealed the lack of sensitivity of ELISA compared with genotypic assays [26].

\section{Polymerase Chain Reaction/Multiplex PCR}

PCR is now gaining acceptability as the method of choice in the detection of Cryptosporidium because of higher sensitivity compared to other methods. Aside from this, it also has the capacity to detect co-enteropathogens (multiplex), to quantify the amount of parasites present in the sample when utilizing quantitative PCR (qPCR), and discriminate between infecting strains [27]. Also, qPCR is sensitive enough to detect exceptionally low copy number of the organism [28]. The simultaneous detection in a single assay provides substantial savings in cost and time in identifying the specific infectious agent and does not require visual determination or antibody binding, thus permitting early and appropriate therapy initiation in a timely and effective manner.

Detection of C. parvum by PCR was first reported in 1991. Since then, several techniques have been developed to detect and differentiate Cryptosporidium at the species and subtype level. Nucleic acid-based methods to screen or detect the presence of Cryptosporidium involves isolating its DNA, combining the isolate with PCR mixture that contains primers and/or probes targeting a region of its nucleic acid sequences, amplifying the target sequence, and detecting the product via gel or fluorescent dyes. Prior to DNA extraction, it is necessary to break open the oocysts. Various methods have been reported such as freeze-thawing, boiling, or use of beads [29]. Amplified products can be further analyzed by restriction enzyme digest or by sequence analysis. This approach has significant advantages due to the flexibility of the primer design. The development of the droplet digital PCR now provides absolute quantification without the need for calibration curves or normalization to reference genes. A recent article reported less affection by inhibitors, lower template copy numbers, but higher costs, when compared with qPCR [30] .

The gene encoding the $18 \mathrm{~S}$ rRNA region is being used more frequently as the target [31] because of more studies reporting a higher sensitivity in relation to its copy number, although other genes have also been reported, such as Cryptosporidium oocyst wall protein [32], 60-kDa glycoprotein, heat shock protein 70, Laxer locus, and microsatellite loci $[33,34]$. Discrimination of species utilizes sequencing because of the very close similarity.

\section{Loop-Mediated Isothermal Amplification}

Loop-mediated isothermal amplification (LAMP) is an emerging technology and recognized as a useful diagnostic tool in Cryptosporidium detection [35]. It has major advantages over other diagnostic methods for detection of 
Cryptosporidium especially at relatively low concentration in environmental samples [36]. Comparative detection of LAMP, PCR, and IFA has been documented in several epidemiological studies. In a study in Iran, LAMP showed higher sensitivity than PCR and IFA in the detection of Cryptosporidium oocyst in water samples [37•]. Similarly, a sensitivity assay performed used in a study in Turkey determined that LAMP can detect $C$. parvum DNA at $1.8 \mathrm{fg}$ concentration, as compared to $100 \mathrm{fg}$ by nested PCR [38•]. Development in LAMP assays has improved detection limits for Cryptosporidium in water and fecal samples. LAMP in combination of ARAD filter for the detection of Cryptosporidium oocysts in drinking water detected at least one oocyst in 101 of drinking water [39]. Findings from a study using RT-LAMP targeting 18S sRNA had a detection limit of as low as $6 \times 10^{-3}$ oocysts/test tube using water samples [40]. LAMP has been shown to be highly specific and sensitive for the detection of different Cryptosporidium species using different target genes. In particular, primer sets targeting gp60 was used to detect C. parvum [36], heat shock protein (HSP)-70 for Cryptosporidium andersoni [41], and S-adenosyl-methionine synthetase (SAM) gene for C. parvum, C. hominis, and Cryptosporidium meleagridis in fecal and water samples [38•, 41]. A new approach of an inclusion of a restriction site to generate clonable LAMP products was used to validate findings by sequencing [41]. A commercially available LAMP kit (Loopamp $^{\circledR}$, Eiken Chemical Co, Japan) is now being used for the detection of Cryptosporidium in environmental samples. Although LAMP is rapid and effective method of diagnostic method, one current limitation is the limited range of available sequences of Cryptosporidium species for primer design. Further development of different LAMP methods would lead to better decisions concerning treatment, contamination, and public health risks. Due to its sensitivity and simplicity, this method may prove to be a useful diagnostic tool in epidemiologic studies of Cryptosporidium detection.

\section{Fluorescent In Situ Hybridization}

The epidemiological importance of human cryptosporidiosis resulted in the development of innovative techniques that would identify oocysts in both clinical and environmental samples. Direct identification of zoonotic Cryptosporidium species without the use of PCR-based methods is of great importance during outbreak investigations. Simpler tools that provide species-specific information would enable water utilities and public health authorities to better assess the potential human health risks associated with Cryptosporidium positive environmental samples. One of these techniques is the fluorescence in situ hybridization (FISH). This method utilizes fluorescently labeled complementary DNA oligonucleotide probes that target-specific sequences of cellular rRNA for direct identification of microorganisms. Ribosomal RNA
(rRNA) targeted oligonucleotide probes provide an advantage because they can be designed to various degrees of specificity, reaching from genus to species and even subspecies level. Fluorescently labeled rRNA targeted probes applied in FISH have emerged as a powerful tool for the detection of microorganisms in a wide range of environmental samples. The rRNA is used because it is a naturally amplified target for hybridization probes due to its high copy number. The procedure includes the following steps: (i) fixation of the specimen; (ii) preparation of the sample, including specific pre-treatment steps; (iii) hybridization with the respective probes for detecting respective targets; (iv) washing steps to remove unbound probes; (v) mounting, visualization, and documentation of results [42-44].

The first use of 6-carboxyfluorescein phosphoramidite as a label for fluorescent in situ hybridization for specific detection of C. parvum was developed in 1997 [45]. The probe set consisted of two synthetic oligonucleotides each tagged with a fluorescent reporter molecule. Each probe strand detects ribosomal RNA from a range of isolates of this species, and the combination was designed to allow detection of all isolates. However, the sets were not tested for utility in actual water samples.

A fluorescent in situ hybridization (FISH) technique developed by Vesey et al. (1998) shows considerable promise as an indicator of $C$. parvum oocyst viability [46]. In these assays, a fluorescent DNA probe is targeted to the $18 \mathrm{~S}$ rRNA of C. parvum. The $18 \mathrm{~S}$ rRNA is usually present in viable organisms and is degraded by cellular RNases in dead or dying cells. Hybridization with the designed Cry1 probe resulted in fluorescence of sporozoites within oocysts that were capable of excystation, while oocysts that were dead prior to fixation did not fluoresce. Results also showed that the fluorescence of FISH-stained oocysts was not bright enough to enable detection of oocysts in environmental water concentrates containing auto-fluorescent algae and mineral particles. However, in combination with immunofluorescence staining, FISH enabled species-specific detection and viability determination of $C$. parvum oocysts in water samples. The $18 \mathrm{~S}$ rRNA can be used successfully for species-specific design of probes for FISH detection of Cryptosporidium spp. FISH using a C. parvum-specific probe provided an alternative tool for accurate identification of zoonotic Cryptosporidium which can be applied to both epidemiological and outbreak investigations [43, 46, 47]. Since its development, several studies have utilized FISH in recovering C. parvum in flies which causes mechanical transmission of the pathogen [48], direct and rapid detection of $C$. parvum on polytetrafluoroethylene (PTFE) membrane [49], testing of recreational beach water samples [50], and visualizing C. parvum life-cycle stages in cell-free culture [51].

Although most studies focused on detection of $C$. parvum only, a two-color FISH assay, based on species-specific probes 
for $C$. parvum and $C$. hominis, can distinguish between the two major species involved in human infections. The potential to detect and identify pathogenic Cryptosporidium species in clinical, water, and environmental samples within a 3-h time frame demonstrates that FISH presents an alternative to PCRbased assays [52].

The major drawback for FISH technique, however, is that it is limited to measuring the viability of $C$. parvum oocysts and not their infectivity. The extent to which FISH probes are useful for studies of oocyst viability is dependent on the rate at which SSU rRNA decays. Quantitative assessment of rapid decay upon cell death for SSU rRNA has not really been done, and it is likely that the rate of degradation will vary depending on different environmental conditions (i.e., temperature, $\mathrm{pH}$, salinity, and/or RNase contamination present) [53].

\section{Recent Advances}

\section{Nanotechnology-Based Platforms}

The potential of nanotechnology-based materials has been utilized to improve specificity and sensitivity of other detection methods such as ELISA, PCR, lateral flow assays, and immunofluorescent-antibody microscopy $[1 \bullet, 54 \bullet, 56]$. One of the main problems encountered in IFA microscopy is the presence in water samples of inert particles or algal cells which have strong auto-fluorescence that can compete with the signal of labeled cells. Although fluorescent dyes have been suggested to effectively label oocysts in water samples, they have high susceptibility to photodegradation and have broad excitation and emission spectra. The use of semiconductor quantum dots for immunofluorescent labeling of C. parvum oocysts in water samples has shown to provide excellent and more consistent results [57-59]. Labeling with quantum dots (QD) can detect up to 4495 oocysts. The difference in detected oocysts using quantum dots and FITC does not have any significant difference even at various $C$. parvum oocyst concentrations [58]. In terms of photostability, QDlabeled oocysts exhibit better photostability after being exposed to continuous UV excitation for $5 \mathrm{~min}$ while the intensity of FITC-labeled oocysts can decrease to $19.5 \%$ after the same period [57, 59]. Quantum dot labels also have approximately $50 \%$ lower interference in concentrated water samples compared to organic fluorophore labels [59]. Another application of nanotechnology-based materials for Cryptosporidium diagnosis is the use of oligonucleotide gold nanoparticles for molecular detection without the need for amplification of nucleic acids and proteins [60]. Examples of assays that have been developed using gold nanoparticles are the electrochemical-based sandwich enzyme-linked immunosensor [61], rapid immunodot blot assay [62], and amplification-free detection systems [60] by using a dual- labeled gold nanoparticles (alkaline phosphatase (ALP) and anti-oocyst monoclonal antibody) functionalized indium tin oxide electrode. With this approach, the sensitivity of the electrochemical immunosensor increased with a limit of detection of 3 oocysts $/ \mathrm{ml}$ in a minimal processing period [61].

The other assay developed using gold nanoparticles is the immunodot blot assay. Compared to the conventional method of adding anti-oocyst monoclonal antibodies then ALPconjugated secondary mouse antibodies to immobilized C. parvum, this enhanced method makes use of dual-labeled gold nanoparticles to detect the C. parvum. The sensitivity of the immunodot blot assay was improved by 500 times compared to the conventional method that it can detect as low as 10 oocysts $/ \mathrm{ml}$. The enhanced assay also had good coherence with the results of PCR detection of the organism in water samples [62].

Aside from detection using antibodies, RNA's coding for the 18S rRNA and HSP70 have also been targeted using oligonucleotide gold nanoparticles. The hybridization of nanoparticle probes to $18 \mathrm{~s}$ rRNA, which was done without amplification, was detected without complex technology was able to detect as few as 670 oocysts/ul in spiked stool samples. Cross-reactivity with either $18 \mathrm{~s}$ rRNA of other protozoan parasites or Escherichia coli was not observed [60]. Targeting the HSP70 gene, on the other hand, causes formation of oligonucleotide networks that are visually detected with simple colorimetric readout. Detection after inducing expression of HSP70 has sensitivity that can detect as low as 5000 oocysts [63]. Both of these methods have potential of being used for point-of-care assays for C. parvum.

There are other nanotechnology-based detection methods that have been less studied such as the background-free cytometry with rare earth nanoparticle bioprobes [64], bioimaging with silica-encapsulated europium particles [64], and the use of piezoelectric-excited millimeter-sized cantilever (PEMC) sensors [55, 65].

\section{Towards Point-of-Care Technology}

\section{Lab-on-a-Chip}

The concept of micro total analysis system ( $\mu \mathrm{TAS})$, later indicated as a lab-on-a-chip, was introduced by Manz and colleagues in the early $1990[66,67]$. They introduced the concept of scaling down the size of analytical devices for improved performance and portability. An ideal $\mu \mathrm{TAS}$ requires only a small volume of sample and incorporates all necessary manipulations and analysis steps to deliver a qualitative or, in some cases, quantitative result in a single-in-answer-out manner [68]. The majority of $\mu$ TAS platforms developed for detecting Cryptosporidium are principally for environmental analysis for cryptosporidial contamination from water 
sources. The platforms used for detection range from sizefiltering-based microfluidic devices $[69,70]$ to nucleic acidbased amplification systems [71], the protozoan's dielectrophoretic properties [72, 73], and immunological properties [74]. These $\mu \mathrm{TAS}$ technologies are promising platforms for future point-of-care (POC) platforms that may eventually be suitable not only for environmental surveillance but also as clinical diagnostic tools. One promising technology that has been tested for both epidemiological surveillance and now piloted for clinical diagnosis among HIV patients suspected of cryptosporidiosis is the use of microfluidic immunoassay system targeting the P23 antigen of the Cryptosporidium developed by Zhang and colleagues [75]. The detection process can be completed within 10 min using $2 \mu \mathrm{l}$ of the sample volume. It generated a diagnostic sensitivity of $100 \%$ and diagnostic specificity of $98.2 \%$. Although the technology remains "near" POC, the challenge of miniaturizing the digital conversion of fluorescence signal to a handheld device may well be expected in the near future.

\section{Immunochromatographic Tests}

The development of POC tests for the diagnosis of Cryptosporidium addresses the challenges posed by resource-limited settings and the patient's need for immediate intervention. The majority of POC test kits that have been commercially available over the past decade utilizes the principle of immunochromatographic assays (ICT) in the form of test strips $[56,76,77,78 \bullet, 79,80,81 \bullet, 82 \bullet]$. Generally, the strips follow the lateral flow concept where Cryptosporidium antigen-specific antibodies are bound to a membrane and uses capillary flow to move the labeled antibody-antigen complex [83]. One of the considerations in gauging the performance of a POC test strip is having a turn-around time of $10 \mathrm{~min}-$ the assumed time a microscopist scans a slide prior to declaring negative results $[82 \bullet, 84]$.

Despite its increasing commercial availability and simplicity, a few clinical laboratories in resource-replete regions resort to ICT rapid testing and still consider microscopy as the standard for diagnosis $\left[85,860^{\bullet}\right]$. Aside from a relatively high testing cost, the available POC tests exhibit a wide range of sensitivity, from as low as $13.6 \%[87 \bullet]$ to as high as $100 \%$ $[88 \cdot, 89]$, with a good specificity of $97-100 \%$, nonetheless $[77,80,90,91]$. Due to the wide range of sensitivity, it is necessary to constantly evaluate the tests' diagnostic performance in varying settings with focus on the assessment of its effect on patient-centered outcomes [92•].

Furthermore, the current commercially available POC tests are limited to detecting the presence or absence of the parasite without the capacity to quantify the burden of infection and differentiate between genus [87॰]. However, these limitations can be outweighed if POC tests will be used to accelerate treatment initiation, to test a relatively large number of atrisk patients or to screen individuals during epidemics. Some of the commercial tests comprise of a combination of antibodies specific for $2-3$ antigens such as the RIDA ${ }^{\circledR}$ QUICK Cryptosporidium/Giardia Combi cassettes (R-Biopharm, Germany) [77], ImmunoCard STAT ${ }^{\circledR}$ Crypto/Giardia (Meridian Bioscience, Inc.) [91], and RIDA ${ }^{\circledR}$ QUICK Cryptosporidium/ Giardia/Entamoeba Combi dipsticks (R-Biopharm, Germany) [93•]. These single panels are used to detect an enteric infection caused by either Cryptosporidium or Giardia, as in the case of RIDA $^{\circledR}$ QUICK Cryptosporidium/Giardia Combi and ImmunoCard STAT ${ }^{\circledR}$ Crypto/Giardia, and Cryptosporidium/ Giardia/Entamoeba for the RIDA ${ }^{\circledR}$ QUICK Cryptosporidium/ Giardia/Entamoeba Combi, with comparable performance met-

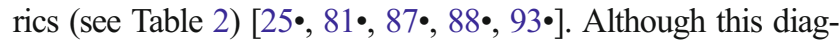
nostic platform is best coupled with standard/routine diagnostic

Table 2 List of common commercially available immunochromatographic tests and their corresponding performance metrics

\begin{tabular}{|c|c|c|c|c|}
\hline ICT test & Sensitivity & Specificity & PPV & NPV \\
\hline Savyon $^{\circledR}$ CoproStrip ${ }^{\mathrm{TM}}[82 \cdot]$ & 74 & 98 & 97 & 94 \\
\hline Coris Duo-Strip ${ }^{\dagger}[88 \bullet]$ & 66.7 & 95.2 & - & - \\
\hline Dia.Pro CA-RT [81•] & 86.7 & 100 & 100 & 95.2 \\
\hline Coris Crypto-Strip [77, 91] & 61.1 & 99.3 & 80 & 98 \\
\hline Techlab Quik Chek $[88 \bullet, 89]$ & 100 & 100 & 100 & 100 \\
\hline Meridian ImmunoCard STAT! ${ }^{\circledR}$ CGE $^{\ddagger}\left[88^{\bullet}\right]$ & 100 & 95.4 & - & - \\
\hline Meridian ImmunoCard STAT! ${ }^{\circledR} \mathrm{CG}^{\dagger}[25 \bullet, 81 \cdot, 91]$ & 87.3 & 99.6 & 95.6 & 98.6 \\
\hline ThermoFisher Remel ${ }^{\mathrm{TM}}$ Xpect $^{\mathrm{TM}}[91]$ & 68.8 & 100 & - & - \\
\hline R-Biopharm RIDA ${ }^{\circledR}$ QUICK Cryptosporidium $[23,77,88 \bullet, 90,93 \bullet]$ & 83.1 & 98.6 & 89.4 & 97.8 \\
\hline R-Biopharm RIDA ${ }^{\circledR}$ QUICK CG Combi ${ }^{\dagger}[77,87 \bullet, 93 •]$ & 53.4 & 98.9 & 91.7 & 82.6 \\
\hline R-Biopharm RIDA ${ }^{\circledR}$ QUICK CGE Combi ${ }^{\ddagger}[93 \bullet]$ & 71.1 & 94.3 & 65.2 & 95.6 \\
\hline
\end{tabular}

${ }^{\dagger}$ A single platform that simultaneously detects Cryptosporidium and Giardia infections.

\$ A single platform that simultaneously detects Cryptosporidium, Giardia and Entameoba infections. 
procedures, it can be a primary option for the rapid qualitative diagnosis of a possible enteric parasite for providing immediate intervention or a basis for performing further laboratory testing.

\section{Conclusion}

While the challenge of therapeutic intervention for Cryptosporidium remains a huge task, the challenge of diagnostic technology for active case detection has moved at considerable speed. The major limiting factor of these existing technologies is access. The major driver for developing diagnostic point-of-care testing is to improve the turn-around-time of such testing, thereby allowing clinicians and patients to make a quick clinical decision. For point-of-care tests to have maximal impact in resource-limited settings, it is necessary for the cost of the technology to be affordable even to the marginalized sector of society. To optimize point-of-care testing in resource-limited settings, diagnostic tests need rigorous assessments focused on relevant clinical outcomes and operational costs, which differ from assessments of conventional diagnostic tests [92•]. The development of a fully integrated POC platform for the diagnosis of Cryptosporidium must include each aspect of test performance such as sample preparation, on-chip nucleic acid analysis and immunoassay, and system integration/automation. At this point in time, ICT platforms remain to be the closest to bedside; however, a challenge remains on the ability to identify and discriminate intensity disease burden. This can be augmented with strong clinical correlation and the knowledge on the burden and epidemiology of the disease in clinical setting.

\section{Compliance with Ethics Guidelines}

Conflict of Interest The authors declare that they have no competing interests.

Human and Animal Rights and Informed Consent This article does not contain any studies with human or animal subjects performed by any of the authors.

\section{References}

Papers of particular interest, published recently, have been highlighted as:

- Of importance

1. Checkley W, White a C, Jaganath D, Arrowood MJ, Chalmers RM, Chen X-M, et al. A review of the global burden, novel diagnostics, therapeutics, and vaccine targets for cryptosporidium. Lancet Infect Dis. 2015;15:85-94. This paper contains the most recent and comprehensive review on the global burden of the disease.
This review also highlighted major gaps in optimum diagnosis, treatment and immunization.

2. Molbak K, Andersen M, Aaby P, Hojlyng N, Jakobsen M, Sodemann $\mathrm{M}$, et al. Cryptosporidium infection in infancy as a cause of malnutrition: a community study from Guinea-Bissau, West Africa. Am J Clin Nutr. 1997;65:149-52.

3. Ochoa TJ, Salazar-Lindo E, Cleary TG. Management of children with infection-associated persistent diarrhea. Semin Pediatr Infect Dis. 2004;15:229-36.

4. Jokipii L, Jokipii AM. Timing of symptoms and oocyst excretion in human cryptosporidiosis. N Engl J Med. 1986;315:1643-7.

5. Chappell CL, Okhuysen PC, Langer-Curry R, Widmer G, Akiyoshi DE, Tanriverdi S, et al. Cryptosporidium hominis: experimental challenge of healthy adults. Am J Trop Med Hyg. 2006;75:851-7.

6. Mac Kenzie WR, Hoxie NJ, Proctor ME, Gradus MS, Blair KA, Peterson DE, et al. A massive outbreak in Milwaukee of cryptosporidium infection transmitted through the public water supply. $\mathrm{N}$ Engl J Med. 1994;331:161-7.

7. Dillingham RA, Lima AA, Guerrant RL. Cryptosporidiosis: epidemiology and impact. Microbes Infect. 2002;4:1059-66.

8. Petersen C. Cryptosporidium and the food supply. Lancet. 1995;345:1128-9.

9. Funari E, Manganelli M, Sinisi L. Impact of climate change on waterborne diseases. Ann Ist Super Sanita. 2012;48:473-87.

10. Putignani L, Menichella D. Global distribution, public health and clinical impact of the protozoan pathogen cryptosporidium. Interdiscip Perspect Infect Dis. 2010;2010.

11 • Shirley D-AT, Moonah SN, Kotloff KL. Burden of disease from cryptosporidiosis. Curr Opin Infect Dis. 2012;25:555-63. This research article provides recent data about the strain and the burden of cryptosporidiosis along with the developments in therapeutic and preventive strategies.

12. Mor SM, Tumwine JK, Ndeezi G, Srinivasan MG, Kaddumulindwa DH, Tzipori S, et al. Respiratory cryptosporidiosis in HIV-seronegative children, Uganda: potential for respiratory transmission. Clin Infect Dis. 2010;50:1366-72.

13. Mohamed AMA, Ahmed MA, Zaglool DA, Ahmed SA-E-G. Molecular evaluation of conventional microscopic method versus fecal antigen capture enzyme-linked immunosorbent assay and rapid immunochromatographic assay for diagnosis of Cryptosporidium infection. Infect Dis Clin Pract. 2015;23(1):26-31.

14. Priest JW, Kwon JP, Moss DM, Roberts JM, Arrowood MJ, Dworkin MS, et al. Detection by enzyme immunoassay of serum immunoglobulin $\mathrm{G}$ antibodies that recognize specific Cryptosporidium parvum antigens. J Clin Microbiol. 1999;37: 1385-92. The study aimed to investigate the frequency of Cryptosporidium infection among suspected versus nonsuspected patients and to evaluate the performances of conventional microscopic assay, Cryptosporidium antigen detection enzyme-linked immunosorbent assay, Crypto-Antigen Rapid Test, for diagnosis of Cryptosporidium infection using Cryptosporidium-specific polymerase chain reaction.

15. Frost FJ, de la Cruz AA, Moss DM, Curry M, Calderon RL. Comparisons of ELISA and Western blot assays for detection of Cryptosporidium antibody. Epidemiol Infect. 1998;121:205-11.

16. Elwin K, Chalmers RM, Hadfield SJ, Hughes S, Hesketh LM, Rothburn MM, et al. Serological responses to Cryptosporidium in human populations living in areas reporting high and low incidences of symptomatic cryptosporidiosis. Clin Microbiol Infect. 2007;13:1179-85.

17. Toyoguchi A, Omata Y, Koyama T, Kamiyoshi T, Furuoka H, Kobayashi Y, et al. Specific IgA antibody response to coproantigens of Cryptosporidium parvum in serum and saliva of calves after experimental infection. Vet Parasitol. 2001;96:213-20.

18. Farkas K, Plutzer J, Moltchanova E, Török A, Varró MJ, Domokos $\mathrm{K}$, et al. Serological responses to Cryptosporidium antigens in 
inhabitants of Hungary using conventionally filtered surface water and riverbank filtered drinking water. Epidemiol Infect. 2015;20:15. This study shows the consistency of IgG antibody responses to act as a reliable markers of exposure.

19. Isaac-Renton JL. Cryptosporidium Serology in Human Populations [Project \#438] [Internet]. 2003 [cited 2015 Apr 8]. Available from: http://www.waterrf.org/ExecutiveSummaryLibrary/90915F_438_ profile.pdf

20. Ware MW, Keely SP, Villegas EN. Development and evaluation of an off-the-slide genotyping technique for identifying Giardia cysts and Cryptosporidium oocysts directly from US EPA Method 1623 slides. J Appl Microbiol. 2013;115:298-309.

21. Keserue HA, Füchslin HP, Wittwer M, Nguyen-Viet H, Nguyen TT, Surinkul N, et al. Comparison of rapid methods for detection of Giardia spp. and Cryptosporidium spp. (Oo)cysts using transportable instrumentation in a field deployment. Environ Sci Technol. 2012;46:8952-9. This study shows how the staining process can affect results of detection of oocysts in fecal samples.

22. Paul S, Chandra D, Tewari AK, Banerjee PS, Ray DD, Boral R, et al. Comparative evaluation and economic assessment of coprological diagnostic methods and PCR for detection of Cryptosporidium spp. in bovines. Vet Parasitol. 2009;164:291-5.

23. Chalmers RM, Campbell BM, Crouch N, Charlett A, Davies AP. Comparison of diagnostic sensitivity and specificity of seven Cryptosporidium assays used in the UK. J Med Microbiol. 2011;60:1598-604.

24. Morgan UM, Pallant L, Dwyer BW, Forbes DA, Rich G, Thompson RC a. Comparison of PCR and microscopy for detection of Cryptosporidium parvum in human fecal specimens: clinical trial. J Clin Microbiol. 1998;36:995-8.

25. Elsafi SH, Al-Maqati TN, Hussein MI, Adam AA, Hassan MMA, Al Zahrani EM. Comparison of microscopy, rapid immunoassay, and molecular techniques for the detection of Giardia lamblia and Cryptosporidium parvum. Parasitol Res. 2013;112:1641-6. The study compares microscopy with other methods to detect oocysts.

26. Nair P, Mohamed JA, DuPont HL, Figueroa JF, Carlin LG, Jiang ZD, et al. Epidemiology of cryptosporidiosis in North American travelers to Mexico. Am J Trop Med Hyg. 2008;79:210-4.

27. Van Lieshout LRM. Clinical consequences of new diagnostic tools for intestinal parasites. Clin Microbiol Infect. 2015;21:520-8.

28. Jothikumar N, da Silva AJ, Moura I, Qvarnstrom Y, Hill VR. Detection and differentiation of Cryptosporidium hominis and Cryptosporidium parvum by dual TaqMan assays. J Med Microbiol. 2008;57:1099-105.

29. Lindergard G, Nydam DV, Wade SE, Schaaf SL, Mohammed HO. The sensitivity of PCR detection of Cryptosporidium oocysts in fecal samples using two DNA extraction methods. Mol Diagn. 2003;7:147-53.

30. Yang R, Paparini A, Monis P, Ryan U. Comparison of nextgeneration droplet digital PCR (ddPCR) with quantitative PCR (qPCR) for enumeration of Cryptosporidium oocysts in faecal samples. Int J Parasitol. 2014;44:1105-13.

31. Nichols RAB, Campbell BM, Smith H V. Identification of Cryptosporidium spp. oocysts in United Kingdom noncarbonated natural mineral waters and drinking waters by using a modified nested PCR-restriction fragment length polymorphism assay. Appl Environ Microbiol. 2003;69:4183-9.

32. Spano F, Putignani L, McLauchlin J, Casemore DP, Crisanti A. PCR-RFLP analysis of the Cryptosporidium oocyst wall protein (COWP) gene discriminates between C. wrairi and C. parvum, and between C. parvum isolates of human and animal origin. FEMS Microbiol Lett. 1997;150:209-17.

33. Skotarczak B. Progress in the molecular methods for the detection and genetic characterization of Cryptosporidium in water samples. Ann Agric Environ Med. 2010;17:1-8.
34. Mary C, Chapey E, Dutoit E, Guyot K, Hasseine L, Jeddi F, et al. Multicentric evaluation of a new real-time PCR assay for quantification of Cryptosporidium spp. and identification of Cryptosporidium parvum and Cryptosporidium hominis. J Clin Microbiol. 2013;51:2556-63.

35. Karanis P, Ongerth J. LAMP - a powerful and flexible tool for monitoring microbial pathogens. Trends Parasitol. 2009;25:498-9.

36. Karanis $\mathrm{P}$, Thekisoe $\mathrm{O}$, Kiouptsi $\mathrm{K}$, Ongerth J, Igarashi I, Inoue N. Development and preliminary evaluation of a loop-mediated isothermal amplification procedure for sensitive detection of Cryptosporidium oocysts in fecal and water samples. Appl Environ Microbiol. 2007;73:5660-2.

37. Mahmoudi MR, Kazemi B, Mohammadiha A, Mirzaei A, Karanis P. Detection of Cryptosporidium and Giardia (oo)cysts by IFA, PCR and LAMP in surface water from Rasht, Iran. Trans R Soc Trop Med Hyg. 2013;107:511-7. The paper provides recent data on Cryptosporidium using LAMP and described the advantages of LAMP method compared to other diagnostic tests (PCR and IFA) for Cryptosporidium detection.

38. Koloren Z, Kaya D, Avsar C, Arts F. Detection of Cryptosporidium species in the sea and tap water samples of Black Sea Turkey. J Parasitol. 2013;99:554-7. The paper described the advantages of LAMP method compared to other diagnostic test (Microscopy and Nested PCR) for Cryptosporidium detection.

39. Karanis P. Combination of ARAD microfibre filtration and LAMP methodology for simple, rapid and cost-effective detection of human pathogenic Giardia duodenalis and Cryptosporidium spp. in drinking water. Lett Appl Microbiol. 2010;50:82-8.

40. Inomata A, Kishida N, Momoda T, Akiba M, Izumiyama S, Yagita $\mathrm{K}$, et al. Development and evaluation of a reverse transcriptionloop-mediated isothermal amplification assay for rapid and highsensitive detection of Cryptosporidium in water samples. Water Sci Technol. 2009;60:2167-72.

41. Bakheit MA, Torra D, Palomino LA, Thekisoe OMM, Mbati PA, Ongerth J, et al. Sensitive and specific detection of Cryptosporidium species in PCR-negative samples by loopmediated isothermal DNA amplification and confirmation of generated LAMP products by sequencing. Vet Parasitol. 2008;158:1122.

42. Dorsch M, Veal DA. Detection of Cryptosporidium spp. via Fluorescent In situ Hybridization: Novel Approaches of Background Reduction and Signal Enhancement. In: Thomson RCA, Armson A, Ryan UM, editors. Cryptosporidium From Mol. to Dis. Elsevier B.V. 2003. p. 321-3.

43. Alagappan A, Tujula NA, Power M, Ferguson CM, Bergquist PL, Ferrari BC. Development of fluorescent in situ hybridization for Cryptosporidium detection reveals zoonotic and anthroponotic transmission of sporadic cryptosporidiosis in Sydney. J Microbiol Methods. 2008:75:535-9.

44. Moter A, Göbel UB. Fluorescence in situ hybridization (FISH) for direct visualization of microorganisms. J Microbiol Methods. 2000;41:85-112.

45. Lindquist HDA. Probes for the specific detection of Cryptosporidium parvum. Water Res. 1997;31:2668-71.

46. Vesey G, Ashbolt N, Fricker EJ, Deere D, Williams KL, Veal DA, et al. The use of a ribosomal RNA targeted oligonucleotide probe for fluorescent labelling of viable Cryptosporidium parvum oocysts. J Appl Microbiol. 1998;85:429-40.

47. Quintero-Betancourt W, Peele ER, Rose JB. Cryptosporidium parvum and Cyclospora cayetanensis: a review of laboratory methods for detection of these waterborne parasites. J Microbiol Methods. 2002;49:209-24.

48. Szostakowska B, Kruminis-Lozowska W, Racewicz M, Knight R, Tamang L, Myjak P, et al. Cryptosporidium parvum and Giardia lamblia recovered from flies on a cattle farm and in a landfill. Appl Environ Microbiol. 2004;70:3742-4. 
49. Taguchi T, Shinozaki Y, Takeyama H, Haraguchi S, Yoshino M, Kaneko M, et al. Direct counting of Cryptosporidium parvum oocysts using fluorescence in situ hybridization on a membrane filter. J Microbiol Methods. 2006;67:373-80.

50. Sunderland D, Graczyk TK, Tamang L, Breysse PN. Impact of bathers on levels of Cryptosporidium parvum oocysts and Giardia lamblia cysts in recreational beach waters. Water Res. 2007;41: 3483-9.

51. Boxell A, Hijjawi N, Monis P, Ryan U. Comparison of various staining methods for the detection of Cryptosporidium in cell-free culture. Exp Parasitol. 2008;120:67-72.

52. Alagappan A, Bergquist PL, Ferrari BC. Development of a twocolor fluorescence in situ hybridization technique for species-level identification of human-infectious Cryptosporidium spp. Appl Environ Microbiol. 2009;75:5996-8.

53. Jex AR, Smith HV, Monis PT, Campbell BE, Gasser RB. Cryptosporidium - biotechnological advances in the detection, diagnosis and analysis of genetic variation. Biotechnol Adv. 2008;26: 304-17.

54. Il CH, Chung BS, Ma DW, La GB, Cho SH, Ji MJ, et al. Development of a diagnostic kit to detect Cryptosporidium parvum and Giardia lamblia. Osong Public Heal Res Perspect. 2013;4:14651. This study shows the application of nanotechnology-based particles in the development of a point-of-care diagnostic kit.

55. Xu S, Mutharasan R. Detection of Cryptosporidium parvum in buffer and in complex matrix using PEMC sensors at 5oocystsmL-1. Anal Chim Acta. 2010;669:81-6.

56. Garcia LS, Shimizu RY, Novak S, Carroll M, Chan F. Commercial assay for detection of Giardia lamblia and Cryptosporidium parvum antigens in human fecal specimens by rapid solid-phase qualitative immunochromatography. J Clin Microbiol. 2003;41:209-12.

57. Zhu L, Ang S, Liu WT. Quantum dots as a novel immunofluorescent detection system for Cryptosporidium parvum and Giardia lamblia. Appl Environ Microbiol. 2004;70:597-8.

58. Lee LY, Ong SL, Hu JY, Ng WJ, Feng Y, Tan X, et al. Use of semiconductor quantum dots for photostable immunofluorescence labeling of Cryptosporidium parvum. Appl Environ Microbiol. 2004;70:5732-6.

59. Lee LY, Hu JY, Ong SL, Ng HY, Wong SW, Feng Y TX. Alternative immunofluorescent labeling of Cryptosporidium parvum in water samples using semiconductor quantum dots. Water Environ Res. 2008;80:725-31.

60. Weigum SE, Castellanos-Gonzalez A, White AC, Richards-Kortum R. Amplification-free detection of Cryptosporidium parvum nucleic acids with the use of DNA/RNA-directed gold nanoparticle assemblies. J Parasitol. 2013;99:923-6.

61. Chinnasamy Thiruppathiraja, Veerappan Saroja, Senthilkumar Kamatchiammal PA and MA. Development of electrochemical based sandwich enzyme linked immunosensor for Cryptosporidium parvum detection in drinking water. J Environ Monit. 2011;13:2782-7.

62. Thiruppathiraja C, Kamatchiammal S, Adaikkappan P, Alagar M. An advanced dual labeled gold nanoparticles probe to detect Cryptosporidium parvum using rapid immuno-dot blot assay. Biosens Bioelectron. 2011;26:4624-7.

63. Javier DJ, Castellanos-Gonzalez A, Weigum SE, White AC, Richards-Kortum R. Oligonucleotide-gold nanoparticle networks for detection of Cryptosporidium parvum heat shock protein 70 mRNA. J Clin Microbiol. 2009;47:4060-6.

64. Tian L, Dai Z, Zhang L, Zhang R, Ye Z, Wu J, Jin D YJ. Preparation and time-gated luminescence bioimaging applications of long wavelength-excited silica-encapsulated europium nanoparticles. Nanoscale. 2012;4:3551-7.

65. Campbell GA, Mutharasan R. Near real-time detection of Cryptosporidium parvum oocyst by IgM-functionalized piezoelectric-excited millimeter-sized cantilever biosensor. Biosens Bioelectron. 2008;23:1039-45.

66. Manz A, Graber N, Widmer HM. Miniaturized total chemical analysis systems: a novel concept for chemical sensing. Sensors Actuators B Chem. 1990;1:244-8.

67. Reyes DR, Iossifidis D, Auroux P-A, Manz A. Micro total analysis systems. 1. Introduction, theory, and technology. Anal Chem. 2002;74:2623-36.

68. Reinholt S, Behrent, Green C, Kalfe ABA, Baeumner AJ. Isolation and amplification of mRNA within a simple microfluidic lab on a chip. Anal Chem. 2014;86:849-56.

69. Zhu L, Zhang Q, Feng H, Ang S, Chau FS, Liu W-T. Filter-based microfluidic device as a platform for immunofluorescent assay of microbial cells. Lab Chip. 2004;4:337-41.

70. Taguchi T, Arakaki A, Takeyama H, Haraguchi S, Yoshino M, Kaneko M, et al. Detection of Cryptosporidium parvum oocysts using a microfluidic device equipped with the SUS micromesh and FITC-labeled antibody. Biotechnol Bioeng. 2007;96:272-80.

71. Esch MB, Locascio LE, Tarlov MJ, Durst R a. Detection of viable Cryptosporidium parvum using DNA-modified liposomes in a microfluidic chip. Anal Chem. 2001;73:2952-8.

72. Jesús-Pérez NM, Lapizco-Encinas BH. Dielectrophoretic monitoring of microorganisms in environmental applications. Electrophoresis. 2011;32:2331-57.

73. Su YH, Tsegaye M, Varhue W, Liao K-T, Abebe LS, Smith JA, et al. Quantitative dielectrophoretic tracking for characterization and separation of persistent subpopulations of Cryptosporidium parvum. Analyst. 2014;139:66-73.

74. Taguchi T, Takeyama H, Matsunaga T. Immuno-capture of Cryptosporidium parvum using micro-well array. Biosens Bioelectron. 2005;20:2276-82.

75. Zhang L, Fu Y, Jing W, Xu Q, Zhao W, Feng M, et al. Rapid microfluidic immunoassay for surveillance and diagnosis of Cryptosporidium infection in human immunodeficiency virusinfected patients. Biomicrofluidics. AIP Publishing; 2015;9: 024114.

76. Garcia LS, Shimizu RY. Detection of Giardia lamblia and Cryptosporidium parvum antigens in human fecal specimens using the ColorPAC combination rapid solid-phase qualitative immunochromatographic assay. J Clin Microbiol. 2000;38: 1267-8.

77. Weitzel T, Dittrich S, Möhl I, Adusu E, Jelinek T. Evaluation of seven commercial antigen detection tests for Giardia and Cryptosporidium in stool samples. Clin Microbiol Infect. 2006;12:656-9.

78. Yansouni CP, Merckx J, Libman MD, Ndao M. Recent advances in clinical parasitology diagnostics. Curr Infect Dis Rep. 2014;16:434. Provides a comparative review of the three recent advances in Crypto diagnostics (i.e. nucleic acid amplification, proteomics and rapid tests).

79. Youn S, Kabir M, Haque R, Petri WA. Evaluation of a screening test for detection of Giardia and Cryptosporidium parasites. J Clin Microbiol. 2009;47:451-2.

80. Christy NC V, Hencke JD, Escueta-De Cadiz A, Nazib F, Von Thien $\mathrm{H}$, Yagita K, et al. Multisite performance evaluation of an enzymelinked immunosorbent assay for detection of Giardia, Cryptosporidium, and Entamoeba histolytica antigens in human stool. J Clin Microbiol. 2012;50:1762-3.

81. Zaglool DAM, Mohamed A, Khodari YAW, Farooq MU. CryptoGiardia antigen rapid test versus conventional modified ZiehlNeelsen acid fast staining method for diagnosis of cryptosporidiosis. Asian Pac J Trop Med. 2013;6:212-5. This is the only paper that evaluates a commercially available rapid diagnostic test for Crypto namely Crypto-Giardia antigen rapid test (CA-RT) by Dia.Pro, against conventional microscopy MZN-AF. 
82. Shimelis T, Tadesse E. Performance evaluation of point-of-care test for detection of Cryptosporidium stool antigen in children and HIV infected adults. Parasit Vectors. 2014;7:227. Evaluation results of a commercially available ICT showed relatively low sensitivity and NPV as it failed to correctly diagnose samples with very low oocyst density.

83. El-Moamly AA-R, El-Sweify MA. ImmunoCard STAT! cartridge antigen detection assay compared to microplate enzyme immunoassay and modified Kinyoun's acid-fast staining technique for detection of Cryptosporidium in fecal specimens. Parasitol Res. 2012;110:1037-41.

84. Weber R, Bryan RT, Bishop HS, Wahlquist SP, Sullivan JJ, Juranek DD. Threshold of detection of Cryptosporidium oocysts in human stool specimens: evidence for low sensitivity of current diagnostic methods. J Clin Microbiol. 1991;29:1323-7.

85. Chalmers RM, Campbell B, Crouch N, Davies AP. Clinical laboratory practices for detection and reporting of Cryptosporidium in community cases of diarrhoea in the United Kingdom, 2008. Euro Surveill. 2010;15(48):pii=19731.

86. Manser M, Granlund M, Edwards H, Saez A, Petersen E, Evengard $\mathrm{B}$, et al. Detection of Cryptosporidium and Giardia in clinical laboratories in Europe - a comparative study. Clin Microbiol Infect. 2014:20:O65-71. Provides a comprehensive comparison and evaluation of the different crypto diagnostic tests used on various European laboratories. Results showed that there are significantly few labs using rapid/POC tests (i.e. strips or cassettes).

87. Helmy YA, Krücken J, Nöckler K, Von Samson-Himmelstjerna G, Zessin KH. Comparison between two commercially available serological tests and polymerase chain reaction in the diagnosis of Cryptosporidium in animals and diarrhoeic children. Parasitol Res. 2014;113:211-6. Provides performance evaluation results showed $100 \%$ specificity and PPV, but very low sensitivity and NPV as compared to the two other tests (as low as $13.6 \%$ sensitivity).
88. Van den Bossche D, Cnops L, Verschueren J, Van Esbroeck M. Comparison of four rapid diagnostic tests, ELISA, microscopy and PCR for the detection of Giardia lamblia, Cryptosporidium spp. and Entamoeba histolytica in feces. J Microbiol Methods. 2015;110:78-84. Latest available article that evaluates performance of a common ICT (Rida QUICK) against EIA and PCR.

89. Minak J, Kabir M, Mahmud I, Liu Y, Liu L, Haque R, et al. Evaluation of rapid antigen point-of-care tests for detection of Giardia and Cryptosporidium species in human fecal specimens. J Clin Microbiol. 2012;50:154-6.

90. Abdel Hameed DM, Elwakil HS, Ahmed MA. A single-step immunochromatographic lateral-flow assay for detection of Giardia lamblia and Cryptosporidium parvum antigens in human fecal samples. J Egypt Soc Parasitol. 2008;38:797-804.

91. Agnamey P, Sarfati C, Pinel C, Rabodoniriina M, Kapel N, Dutoit E, et al. Evaluation of four commercial rapid immunochromatographic assays for detection of Cryptosporidium antigens in stool samples: a blind multicenter trial. J Clin Microbiol. 2011;49:1605-7.

92. Drain PK, Hyle EP, Noubary F, Freedberg K a., Wilson D, Bishai WR, et al. Diagnostic point-of-care tests in resource-limited settings. Lancet Infect Dis. 2014;14:239-49. Extensive review of the importance and benefits of point-of-care testing in resource-limited settings. Also proposes a framework for the systematic assessment and evaluation of commercially available POCs

93. Goñi P, Martín B, Villacampa M, García A, Seral C, Castillo FJ, et al. Evaluation of an immunochromatographic dip strip test for simultaneous detection of Cryptosporidium spp, Giardia duodenalis, and Entamoeba histolytica antigens in human faecal samples. Eur J Clin Microbiol Infect Dis. 2012;31:2077-82. Evaluates a single ICT panel that simultaneously detects three enteric parasites (Crypto, Giardia and Entameoba) against, ELISA, microscopy and PCR. 